\title{
Optical Galton board
}

\author{
D. Bouwmeester, ${ }^{1, *}$ I. Marzoli ${ }^{2, \dagger}$ G. P. Karman, ${ }^{1}$ W. Schleich, ${ }^{2}$ and J. P. Woerdman ${ }^{1}$ \\ ${ }^{1}$ Huygens Laboratory, University of Leiden, P.O. Box 9504, 2300 RA Leiden, The Netherlands \\ ${ }^{2}$ Abteilung für Quantenphysik, Universität Ulm, 89069 Ulm, Germany
}

(Received 25 November 1997; revised manuscript received 24 September 1999; published 14 December 1999)

\begin{abstract}
The conventional Galton board illustrates diffusion in a classical-mechanics context: it is composed of balls performing a random walk on a downward sloping plane with a grid of pins. We introduce a wave-mechanical variety of the Galton board to study the influence of interference on the diffusion. This variety consists of a wave, in our experiments a light wave, propagating through a grid of Landau-Zener crossings. At each crossing neighboring frequency levels are coupled, which leads to spectral diffusion of the initial level populations. The most remarkable feature of the spectral diffusion is that below a certain single-crossing transition probability (around $0.7-0.8$ ) the initial spectral distribution almost perfectly reappears periodically when the wave penetrates further and further into the grid of crossings. We compare our experimental results with numerical simulations and with an analytical description of the system based on a paper by Harmin [Phys. Rev. A 56, 232 (1997)].
\end{abstract}

PACS number(s): $32.80 . \mathrm{Bx}, 32.60 .+\mathrm{i}$

\section{INTRODUCTION}

The classical Galton board is illustrated in Fig. 1(a). Balls are rolling down a sloping board and are scattered by a grid of pins. The random walk performed by the balls leads to Gaussian diffusion. One can think of several quantum- or wave-mechanical varieties of this model by which the influence of interference terms on the diffusion can be studied. We present an optical variety which deals with spectral diffusion of a light wave. The optical Galton board consist of frequency levels inside an optical resonator that are periodically coupled. The coupling is achieved by performing optical Landau-Zener crossings induced by birefringent crystals inside the resonator, as illustrated in Fig. 2 [1]. The resulting level structure of the optical Galton board is shown in Fig. $1(b)$.

Spectral diffusion has been studied extensively in the field of quantum chaos [2,3]. In particular the quantum suppression of spectral diffusion for the kicked quantum rotor received a lot of attention, both theoretically [4] and experimentally [5]. It seems clear that periodic coupling between discrete energy levels and the preservation of coherence between the levels are necessary to obtain suppression; however, a clear understanding of the phenomenon is still missing. In the present context it is worth noting that "quantum chaos" is maybe more aptly called "wave chaos," as illustrated by the study of microwave billiards [6].

Although the optical Galton board is a classical system, we will show that it can be described by a Schrödinger equation and that it fulfills the requirements of periodic coupling between discrete levels and of preserving coherence. Whereas for the kicked quantum rotor the energy-level spac-

*Present address: University of Oxford, Clarendon Laboratory, Parks Road, Oxford OX1 3PU, United Kingdom.

${ }^{\dagger}$ Present address: Dipartimento di Matematica e Fisica and INFM, Università degli Studi di Camerino, Via Madonna delle Carceri, 62032 Camerino (MC), Italy. ing is proportional to $(2 n+1)$, where $n$ is the quantum number of the lower level, the energy- or frequency-level spacing of the optical Galton board is constant. A proper understanding of this most simple model for spectral diffusion seems useful for a clear understanding of the diffusion properties of more complicated systems such as the kicked quantum rotor. We show experimentally that for the optical Galton board suppression of diffusion can occur in the form of almost perfect recurrences of the initial level population. But, as we

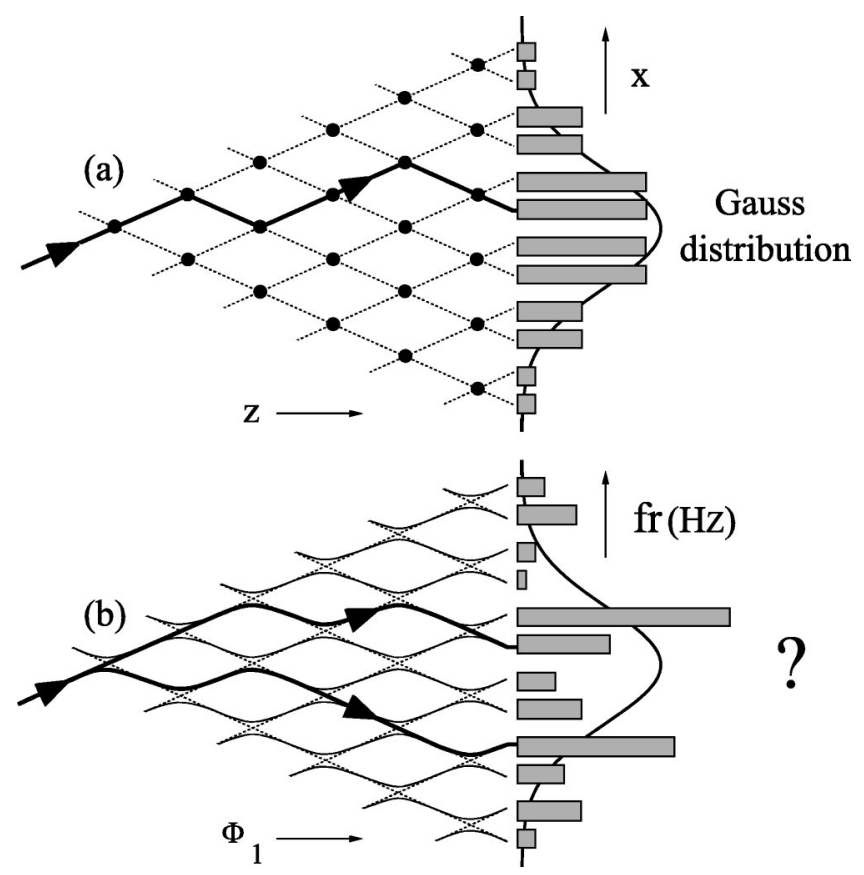

FIG. 1. (a) The classical Galton board, sloping downwards in the $z$ direction, yields a Gaussian distribution function for the final position of the particle along the $x$ axis. The dots represent pins at which the balls are scattered. (b) The grid of Landau-Zener crossings which is the quantum- or wave-mechanical analog of the classical Galton board; $f r$ indicates the frequency axis, and $\phi_{1}$ is a control parameter which is proportional to time. 

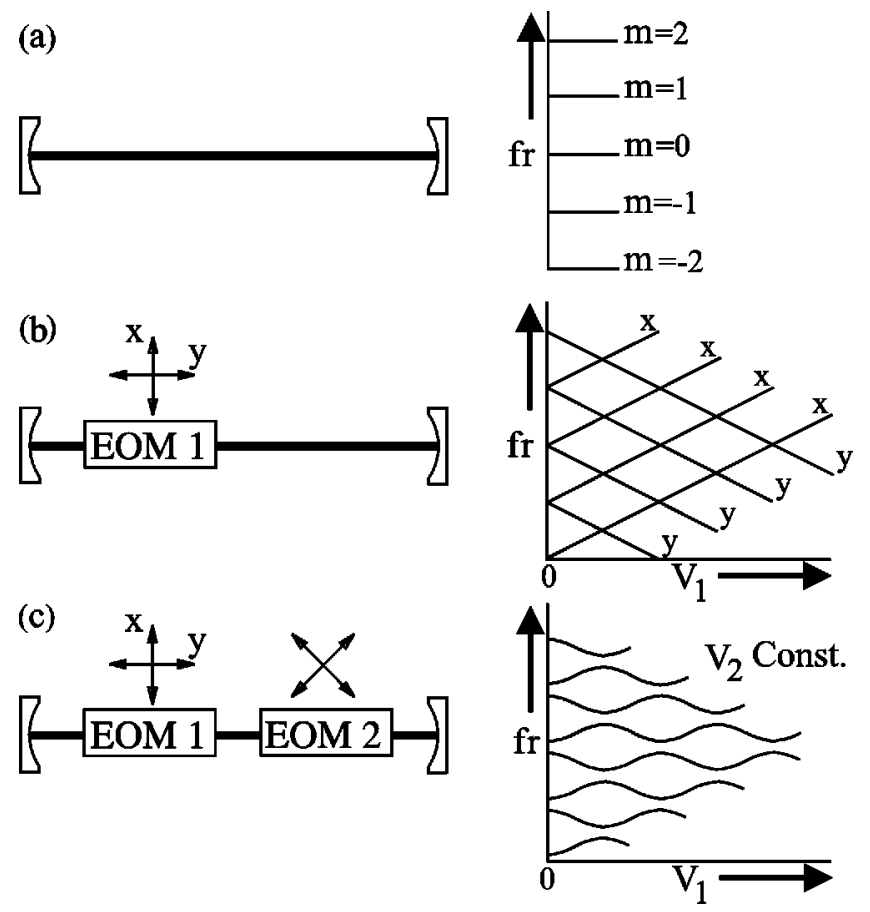

FIG. 2. (a) Sketch of a linear optical resonator which has equidistant longitudinal modes $(m=0, \pm 1, \pm 2)$. (b) Including a electro-optic modulator (EOM1) inside the resonator, and increasing the voltage $V_{1}$ across the modulator, leads to crossing levels with orthogonal polarizations $x$ and $y$. (c) Including a second modulator (EOM2) inside the resonator, rotated over $45^{\circ}$ with respected to the optical axis of EOM1 and with a constant applied voltage $\left(V_{2}\right)$, turns each level crossing into an avoided crossing (LandauZener crossing).

will see, even for the optical Galton board the diffusion properties turn out to be rather complicated and some features still lack a qualitative understanding.

Since for the optical Galton board the number of populated levels increases at each step into the grid of crossings, the amplitudes in the outermost levels can never interfere and are in general nonzero. Therefore, the observed recurrences of the initial level population can never be perfect and it is not obvious that "imperfect" recurrences should be expected. In fact, whether or not recurrences are present depends on the transition probability at the avoided crossing, as shown theoretically by Harmin [7]. Above a certain transition probability, around $0.7-0.8$, the recurrences cease to exist! In a recent paper by Törmä it was suggested that this change in dynamics is similar to a phase transition in the Ising model [8]. Beside its relation to the study of quantum or wave diffusion, the optical Galton board could be of practical interest to the study of selective-field ionization of Rydberg atoms where strong Stark splitting of the Rydberg levels creates similar grids of Landau-Zener crossings $[9,10]$.

In Sec. II we present the model for the optical Galton board. The experimental set up is presented in Sec. III. The experimental results together with the numerical calculations, based on the theoretical model, are collected in Secs. IV and V. In Sec. VI we give a brief review of the theory by Harmin on quantum diffusion on a grid of Landau-Zener crossings [7], and compare our results with theory. A concluding discussion is given in Sec. VII.

\section{MODEL OF THE OPTICAL GALTON BOARD}

The basic idea of the optical Galton board is as follows. Consider a linear optical resonator which has a ladder of equidistant resonant frequencies, as illustrated in Fig. 2(a). We restrict our attention to the longitudinal cavity modes (labeled with $m=0, \pm 1, \pm 2, \ldots$, where $m$ is the mode index relative to that of an arbitrarily defined "center" mode). Each mode is polarization independent, i.e., each mode has a two-fold polarization degeneracy. This polarization degeneracy is lifted by inserting a birefringent crystal, in the form of an electro-optical modulator (EOM1), into the resonator. Increasing the birefringence as function of time, which can be done by applying an increasing electric voltage $\left(V_{1}\right)$ across EOM1, results in two crossing manifolds of levels with orthogonal polarizations, $x$ and $y$, as shown in Fig. 2(b). A second birefringent crystal (EOM2) is placed inside the resonator with its axes rotated $45^{\circ}$ with respect to the axes of the first crystal. A constant voltage across EOM2 provides a coupling between the crossing levels of orthogonal polarization and turns each level crossing into an avoided (LandauZener) crossing; see Fig. 2(c). The initial state of the optical Galton board is prepared by injecting laser light at one specific resonant frequency and polarization into the resonator. As soon as the intensity inside the resonator exceeds a certain value, the injection laser is switched off and the voltage across EOM1 is linearly increased while the voltage across EOM2 is kept constant. In this way the initial mode population will be coupled via the avoided crossings to more and more modes, which results in a coherent diffusion process. This spectral diffusion can be monitored by analyzing the small fraction of light that is leaking out of the resonator through one of the mirrors.

We now employ the Jones-matrix formalism to present the optical Galton board in a more formal way $[1,11-13]$. In this formalism the polarization of the population in a single cavity mode is represented by a 2 vector $\vec{E}(t)=(x(t), y(t))$, where $x(t)$ and $y(t)$ are slowly varying (with respect to the optical frequency) amplitudes of the $x$ and $y$ polarization components. Each polarization-changing optical element inside the resonator is represented by a $2 \times 2$ matrix which acts on $\vec{E}(t)$. EOM1 and EOM2 are represented by

$$
\begin{gathered}
B_{1}(t)=\left(\begin{array}{cc}
e^{i \phi_{1} / 2} & 0 \\
0 & e^{-i \phi_{1} / 2}
\end{array}\right), \\
B_{2}(t)=\left(\begin{array}{cc}
\cos \left(\phi_{2} / 2\right) & -i \sin \left(\phi_{2} / 2\right) \\
-i \sin \left(\phi_{2} / 2\right) & \cos \left(\phi_{2} / 2\right)
\end{array}\right),
\end{gathered}
$$

respectively, where $\phi_{1}$ and $\phi_{2}$ are the phase differences which the two orthogonal polarizations along the axis of birefringence obtain by passing EOM1 and EOM2. One can construct a round-trip matrix $M(t)$ by multiplying the matrices representing all the optical elements that the light passes during one round trip. In the case of the optical Galton board 
there are two EOM's which are passed twice for each round trip in the linear cavity. Neglecting optical losses, the round-trip matrix is given by

$$
M(t)=B_{2}(t) B_{1}(t) B_{1}(t) B_{2}(t)=\left(\begin{array}{cc}
-i \sin \left(\phi_{1}\right)+\cos \left(\phi_{1}\right) \cos \left(\phi_{2}\right) & -i \cos \left(\phi_{1}\right) \sin \left(\phi_{2}\right) \\
-i \cos \left(\phi_{1}\right) \sin \left(\phi_{2}\right) & i \sin \left(\phi_{1}\right)+\cos \left(\phi_{1}\right) \cos \left(\phi_{2}\right)
\end{array}\right) .
$$

We assume that the round-trip matrix is approximately constant during a single round-trip time $T$. This implies that the change in birefringence of the EOM's per round trip should be much smaller than $2 \pi$. Under this condition the time evolution of $\vec{E}(t)$ is governed by

$$
\vec{E}(t+T)=M(t) \vec{E}(t) .
$$

The evolution of $\vec{E}(t)$ can be cast in the form of a Schrödinger-like equation

$$
\frac{d \vec{E}}{d t}=-\frac{i}{T} H \vec{E}
$$

where the elements of $H$ are expressed in phase shifts per round-trip time $T$, in anticipation to the relation of $H$ with the round-trip matrix $M$. Equation (4) yields an alternative expression for Eq. (3),

$$
\vec{E}(t+T)=\exp \left\{-\frac{i}{T} \int_{t}^{t+T} H\left(t^{\prime}\right) d t^{\prime}\right\} \vec{E}(t) .
$$

The case in which $H$ is approximately constant during a round-trip time leads to

$$
M(t)=\exp \{-i H(t)\} .
$$

Equations (5) and (6) map the classical optical system onto a quantum-mechanical system. For $M(t)$ given by Eq. (2) we obtain the Hamiltonian

$$
H(t)=\frac{\varphi(t)}{\sin \varphi(t)}\left(\begin{array}{cc}
\sin \left(\phi_{1}\right) & \cos \left(\phi_{1}\right) \sin \left(\phi_{2}\right) \\
\cos \left(\phi_{1}\right) \sin \left(\phi_{2}\right) & -\sin \left(\phi_{1}\right)
\end{array}\right)
$$

where $\varphi(t)=\arccos \left(\cos \left(\phi_{1}\right) \cos \left(\phi_{2}\right)\right)$ [1]. The eigenvalues of $H(t)$ are $\pm \varphi(t)$ and for a constant value of $\phi_{2}$, they are plotted as a function of $\phi_{1}$ in Fig. 3 (thick lines). Note that when $\phi_{1}=\alpha t$ and $\phi_{2}=\Delta$, with $\phi_{1}(\bmod 2 \pi) \ll 2 \pi$ and $\phi_{2}$ $\ll 2 \pi$, the model reduces to the Landau-Zener (LZ) Hamiltonian $[14,15]$ given by

$$
H_{\mathrm{LZ}}=\left(\begin{array}{cc}
\alpha t & \Delta \\
\Delta & -\alpha t
\end{array}\right)
$$

These conditions are fulfilled in the neighborhood of each avoided crossing, as shown in the dashed square in Fig. 3. In Secs. IV and V we will use the parameters $\alpha$ and $\Delta$ to characterize the settings of the optical Galton board.

For the following discussion it is convenient to introduce the parameter

$$
\tan \theta=-\frac{\cos \left(\phi_{1}\right) \sin \left(\phi_{2}\right)}{\sin \left(\phi_{1}\right)}
$$

from which one can derive $\dot{\theta} / 2$, which is the adiabatic coupling strength between the two frequency levels with orthogonal polarizations [1].

A description of a regular grid of Landau-Zener crossings is obtained by superimposing the two-level structure to a ladder of equally spaced angular frequency levels. As illustrated above in Fig. 2, an optical resonator has intrinsically such a multilevel structure with spacing $\omega_{\mathrm{FSR}}$ equal to $2 \pi$ times the free spectral range $f_{\mathrm{FSR}}=c / 2 \mathrm{~L}$, where $c$ is the velocity of light and $\mathrm{L}$ is the length of the linear resonator. We label the polarization amplitudes of the two levels for each longitudinal mode $m$ by $x_{m}$ and $y_{m}$. The full time evolution for these amplitudes, including the coupling between all levels, can be found in Ref. [13]. The complete model can, however, be simplified under the assumption that coupling is restricted to the neighboring levels only (arrow 2 in Fig. 3 indicates such a coupling). This amounts to neglecting the influence of fast rotating terms, i.e. the coupling between levels separated in angular frequency by at least $\omega_{\mathrm{FSR}}$ (arrows 1 and 3 in Fig. 3 indicate such couplings). Under our experimental conditions (see Sec. III), such an approxima-

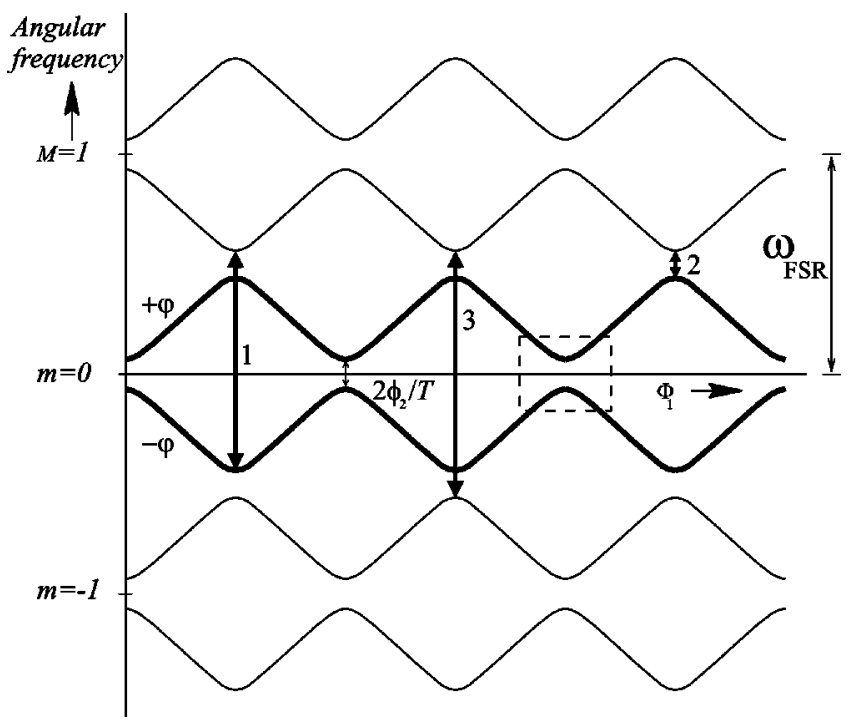

FIG. 3. Adiabatic levels of the optical Galton board. The thick lines indicate a single two-level system. In our optical experiment we make use of a ladder of such two-level systems which are equally spaced by the $\omega_{\mathrm{FSR}}$ which is $2 \pi$ times the free spectral range. The arrows 1,2 , and 3 , indicate couplings between angularfrequency levels (see text). 


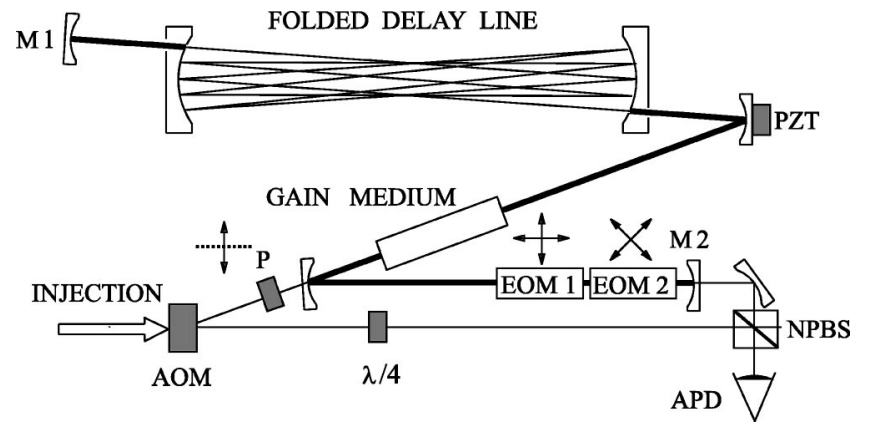

FIG. 4. Schematic drawing of the optical part of the setup. The end mirrors of the resonator are indicated by $M 1$ and $M 2$. The acronyms used are AOM for the acousto-optic modulator, $P$ for the polarizer, EOM for the electro-optic modulator, PZT for the piezo element, NPBS for the nonpolarizing beam splitter, and APD for the avalanche photodiode.

tion seems justified since $\Delta \approx 0.6<(<) 2 \pi[13]$. This approximation results in the following set of equations for the level populations:

$$
\begin{aligned}
\frac{d}{d t} x_{m}(t)= & -i\left(m+\frac{\varphi}{2 \pi}\right) \omega_{\mathrm{FSR}} x_{m}(t) \\
& -\frac{\dot{\theta}}{2} \frac{\sin (\varphi)}{\varphi} y_{m}(t)-\frac{\dot{\theta}}{2} \frac{\sin (\varphi)}{\varphi-\pi} y_{m+1}(t), \\
\frac{d}{d t} y_{m}(t)= & -i\left(m-\frac{\varphi}{2 \pi}\right) \omega_{\mathrm{FSR}} y_{m}(t)+\frac{\dot{\theta}}{2} \frac{\sin (\varphi)}{\varphi} x_{m}(t) \\
& +\frac{\dot{\theta}}{2} \frac{\sin (\varphi)}{\varphi-\pi} x_{m-1}(t) .
\end{aligned}
$$

The first term on the right-hand sides of Eqs. (10) and (11) represents the adiabatic frequency levels, as illustrated in Fig. 3. The second and third term on the right-hand sides describes the coupling between neighboring levels.

\section{EXPERIMENTAL SETUP}

Figure 4 is a schematic drawing of the optical set up that realizes the optical Galton board. $M 1$ and $M 2$ indicate the two end mirrors of the linear optical resonator. Besides the two electro-optical modulators (EOM1 and EOM2), the resonator includes an optical delay line and an optical gain medium. The latter compensates almost completely the optical losses; in fact, the system shown in Fig. 4 is a laser precisely at threshold. The total resonator length is approximately 100 $\mathrm{m}$ which yields a mode spacing of $1.52 \mathrm{MHz}$ and a roundtrip time $T$ of $0.7 \mu \mathrm{s}$. Due to the aperturing by the HeNe gain capillary and the EOMs, the lowest-loss transverse mode of our resonator is the only one that is significantly excited (this has been checked by studying the output spectrum of the system when the gain was set slightly above threshold). One mirror is placed on a piezo element in order to tune one of the longitudinal resonator modes (by definition this is the mode $m=0$ ) to the frequency of the linearly polarized single-frequency $\mathrm{He}-\mathrm{Ne}$ injection laser $(\lambda=633 \mathrm{~nm})$. The light injected into the resonator is the first order deflected beam ( $80 \mathrm{MHz}$ shifted with respect to the zeroth-order beam) from an acousto-optic modulator (AOM). As soon as the intra cavity intensity has built up to about a few $\mathrm{mW}$ the AOM is switched off, hence no more light is injected into the resonator. From that moment on we have the cavity decay time, which was measured to be about $70 \mu \mathrm{s}$, to perform the actual experiment. A linear voltage ramp is applied across EOM1 (typical value for the sweep rate is, $\alpha=3 \times 10^{6} \mathrm{~s}^{-1}$ ), while the voltage applied across EOM2 is kept constant (corresponding typically to $\Delta=0.2 \pi$ for a transition probability around 0.5 ), so that the light inside the resonator passes through the grid of Landau-Zener crossings. After a certain number of steps into the grid the voltage ramp across EOM1 is stopped, and the voltage is fixed at its final value. From that time on the system is in a stationary state so that we can analyze over which levels the light has been distributed.

To determine the final spectral distribution, i.e., the population of the cavity modes after the diffusion, we beat the light leaking out of the resonator through mirror $M 2$ with the zeroth-order beam from the AOM (which is present even if the AOM is switched off). In order to detect both $x$ - and $y$-polarized modes the zeroth-order beam is circularly polarized by using a $\lambda / 4$ wave plate. Measuring in the stationary state a time trace of a few $\mu$ s, using a fast digital oscilloscope (HP 54522A) which can resolve frequencies up to 500 $\mathrm{MHz}$, yields after a Fourier decomposition the spectral distribution. The originally populated frequency level appears in the Fourier decomposition at the AOM frequency of 80 $\mathrm{MHz}$. There are three experimental points which do not influence the main idea of implementing an optical variety of the Galton board but which are of crucial importance for the actual realization.

(i) EOM1 has a maximum range in birefringence corresponding approximately to a $3 \pi$ rad phase shift between $x$ and $y$ polarized light. Since the distance between the avoided crossings corresponds to $\pi \mathrm{rad}$, it seems that a sequence of no more than three crossings can be obtained. To circumvent this limitation we sweep EOM1 back and forth over the interval $[0,2 \pi]$ (see Fig. 5). Each round-trip voltage sweep corresponds to the passage of four Landau-Zener crossings. After the first round-trip voltage sweep, light initially injected at level 0 can be present in levels -3 to 2. In Fig. 5 three out of the 16 possible light trajectories have been drawn by thick lines. After $n$ round-trip voltage sweeps, levels $-(2 n+1)$ to $2 n$ can be populated. This method of populating several levels is similar to the rf excitation scheme for Rydberg atoms [16]. Note that since each crossing is passed twice in succession it should be possible to observe interference effects associated with Stückelberg oscillations $[17,18]$. The main feature of the optical Galton board, i.e., enabling wave-mechanical diffusion over a manifold of levels, is unaffected by this since each double pass will effectively act as a single coupling between neighboring levels.

(ii) The optical losses due to the EOM's and the leakage through the mirrors are compensated for by the gain medium, in our case a polarization-independent He-Ne amplifier tube $(\lambda=633 \mathrm{~nm})$ [12]. Since the amplifying He-Ne medium has a limited bandwidth of approximately $1.5 \mathrm{GHz}$, all 


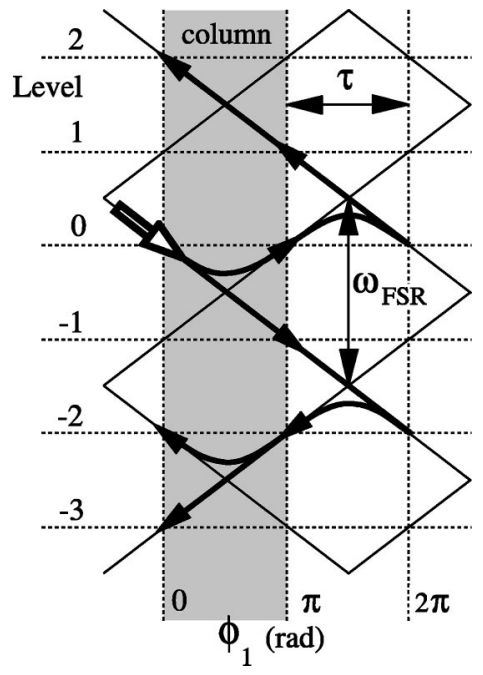

FIG. 5. The thick lines indicate three possible light trajectories, starting from level 0 , which can be traced during the first round-trip voltage sweep. The time it takes to pass a single column (shaded area) of Landau-Zener crossings is, $\tau$, and the angular frequency separation between two subsequent crossings within a column is equal to $\omega_{\mathrm{FSR}}$.

modes accessible via the spectral diffusion should be within $100 \mathrm{MHz}$ around line center in order to have approximately equal $( \pm 5 \%)$ cavity lifetimes. If we restrict the diffusion to about 30 modes, the spacing between them must be less than $3 \mathrm{MHz}$. This is the reason that we use the delay line of approximately $100 \mathrm{~m}$ inside the resonator.

(iii) The photon cavity lifetime obtained in the experiments is approximately $70 \mu \mathrm{s}$, which is about 100 times longer then the cavity round-trip time $T=0.7 \mu \mathrm{s}$. In order to populate sufficiently many modes (say 30 ), some 30 columns in the Landau-Zener grid must be passed. Each LandauZener crossing should therefore be performed within three cavity round-trip times. This condition is in conflict with the assumption in the derivation of Eq. (6) that there are no significant changes in the optical system during a single round-trip time. Experimentally we observed, however, that Eq. (6) remains valid even if a Landau-Zener crossing is performed within a time as short as $3 T$. We will gratefully make use of this property to implement numerical simulations where the dynamics is treated as a succession of twolevel interactions, at each Landau-Zener crossing, followed by adiabatic evolution.

To analyze the experimental results of the following sections it is important to introduce a relative phase, $\Psi$, between crossings within a single Landau-Zener column, i.e., between crossings which form a vertical ladder in Fig. 5. Two subsequent crossings on the ladder are separated in angular frequency by $\omega_{\mathrm{FSR}}$, see Fig. 5; hence the light passing through the higher crossing obtains the additional dynamical phase

$$
\Psi=\tau \omega_{\mathrm{FSR}}
$$

compared to the light passing through the lower crossing. Here $\tau$ is the time it takes to pass a single column.
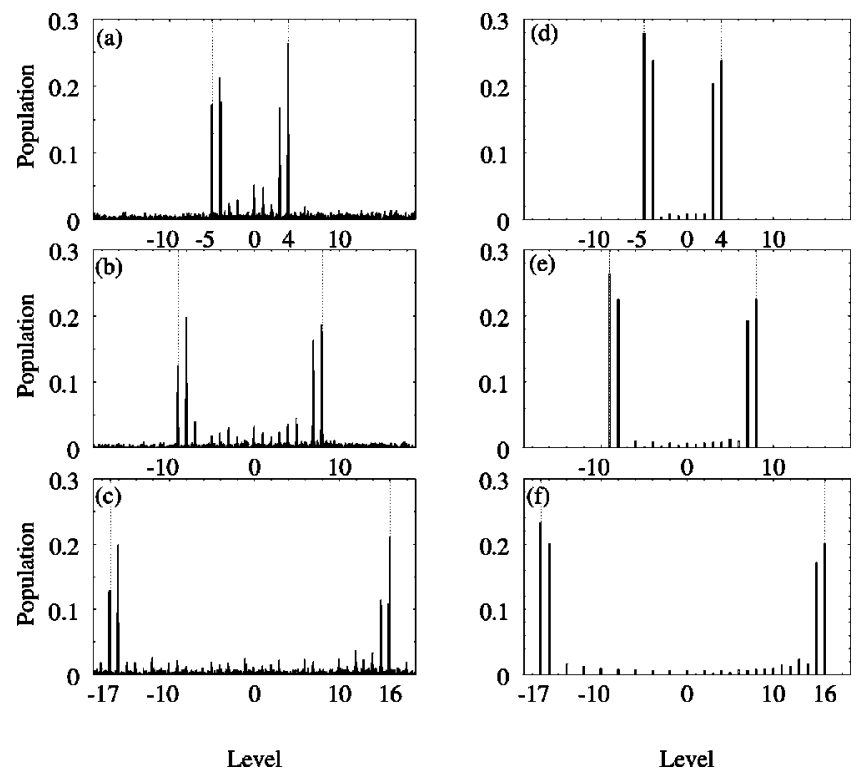

FIG. 6. Experimental test of the coherence quality of the system. After the initial population of level 1 the grid parameters $\alpha$ and $\Delta$ were tuned such that each individual Landau-Zener crossing had a transition amplitude of approximately 0.5 and each double crossing (back and forth through the same crossing as illustrated in Fig. 5) had a transition amplitude close to $1\left[\alpha=3 \times 10^{6} \mathrm{~s}^{-1}, \Delta=(0.2\right.$ $\pm 0.02) \pi]$. The spectral distributions after (a) eight, (b) 16, and (c) 32 columns of crossings are shown. Graphs (d), (e), and (f) show the corresponding numerical results, based on the treatment of the optical Galton board as an array of pointlike beamsplitters in the spectral domain. The dashed lines indicate the outermost levels which can be populated.

\section{COHERENCE QUALITY}

In Sec. III we mentioned the presence of Stückelberg oscillations due to the fact that each crossing is passed twice in succession. If, for example, the transition probability for each crossing is $0.5\left[\alpha=3 \times 10^{6} \mathrm{~s}^{-1}, \Delta=(0.2 \pm 0.02) \pi\right]$, and if the distance between the two successive crossings is tuned properly it should be possible to exchange the populations of the two levels which perform the double crossing. If we initially populate one level and then perform a single crossing followed by a sequence of double crossings and end with another single crossing, it should be possible to observe that only the two outermost levels on each side of the possible range of levels will be populated, provided the grid is tuned properly and coherences are maintained throughout the evolution. The observation of this effect will be a sensitive test for the coherence properties of the optical system. We performed this test experimentally by properly adjusting the coupling strength $\Delta$ and the sweep rate $\alpha$ [see Eq. (8)] to meet the conditions given above. The experimentally obtained distributions after passing eight, 16, and 32 columns of crossings are shown in Figs. 6(a), 6(b), and 6(c), respectively.

Interference completely dominates the dynamics and results in population of the outermost levels in accordance with the theoretical prediction based on coherent evolution. Note that the corresponding incoherent or classical situations 
would yield a Gaussian distribution centered around level $m=0$ [Fig. 1(a)]. We have compared the experimental results with numerical simulations based on the assumption that the optical Galton board is perfectly stable and free of dissipation, and that each avoided crossing can be considered as an isolated Landau-Zener crossings with a transition probability of 0.5 . The simulations take into account the fact that each crossing is passed twice in succession. The result of the simulations are shown in Figs. 6(d), 6(e), and 6(f); they are in reasonable agreement with the experimental results. Since we deal with a complicated interference process, which is sensitive to small changes in the system, the assumptions used in the simulation are too crude to expect perfect agreement between the experiments and the simulation. However, the simulations are adequate for demonstrating the main features of the coherent evolution. The same remark will apply to the numerical results that will be presented in Sec. V. On the basis of the material shown in Fig. 6, we can conclude that the optical system suffers little decoherence and can be used to study coherent diffusion on an optical Galton board.

\section{RECURRENCES}

Whereas in Sec. IV the values of $\alpha$ and $\Delta$ were tuned such that each double crossing resulted in a complete population transfer between each pair of crossing levels, we now consider the case that $\alpha$ and $\Delta$ are tuned such that each double crossing has a transition probability of about 0.5 . This situation constitutes the optical Galton board.

Our main experimental result is shown in Figs. 7(a)-7(d). Figure 7(a) shows the distribution corresponding to the initial state of the optical Galton board. Clearly, only a single mode of the optical system is populated. Figure 7(b) shows how the population has spread out over the neighboring levels after passing eight columns of crossings (effectively four layers of beamsplitters in the spectral domain). The evolution of the populations resembles classical (incoherent) diffusion, and it is only in the large fluctuations in neighboring level populations that the presence of interference can be inferred. Figure 7(c) shows the distribution after 16 columns of crossings. Again there is no evidence that a collective interference effect takes place. Yet we know form the test measurement shown in Sec. IV that coherences are preserved throughout the evolution. Therefore, on first sight, Figs. 7(a) and 7(b) seem to indicate that interferences play a minor role in the diffusion. However, the experimentally obtained distribution after passing 32 columns of crossings, as shown in Fig. 7(d), leads to the complete opposite conclusion. Apparently the initially populated level is almost completely repopulated which can only be the result of some dominant interference effect.

To obtain such a clear recurrence after 32 columns of crossings, we had to tune the parameters of the grid carefully. By changing the grid parameters $\alpha$ and $\Delta$ slightly (so that the transition probability after each double crossing was still between 0.4 and 0.6 ), we could obtain recurrences after any number of columns of crossings, as far as the experimental conditions allow for. Since changes of a few percent in the values of $\alpha$ and $\Delta$ completely change the dynamics of the
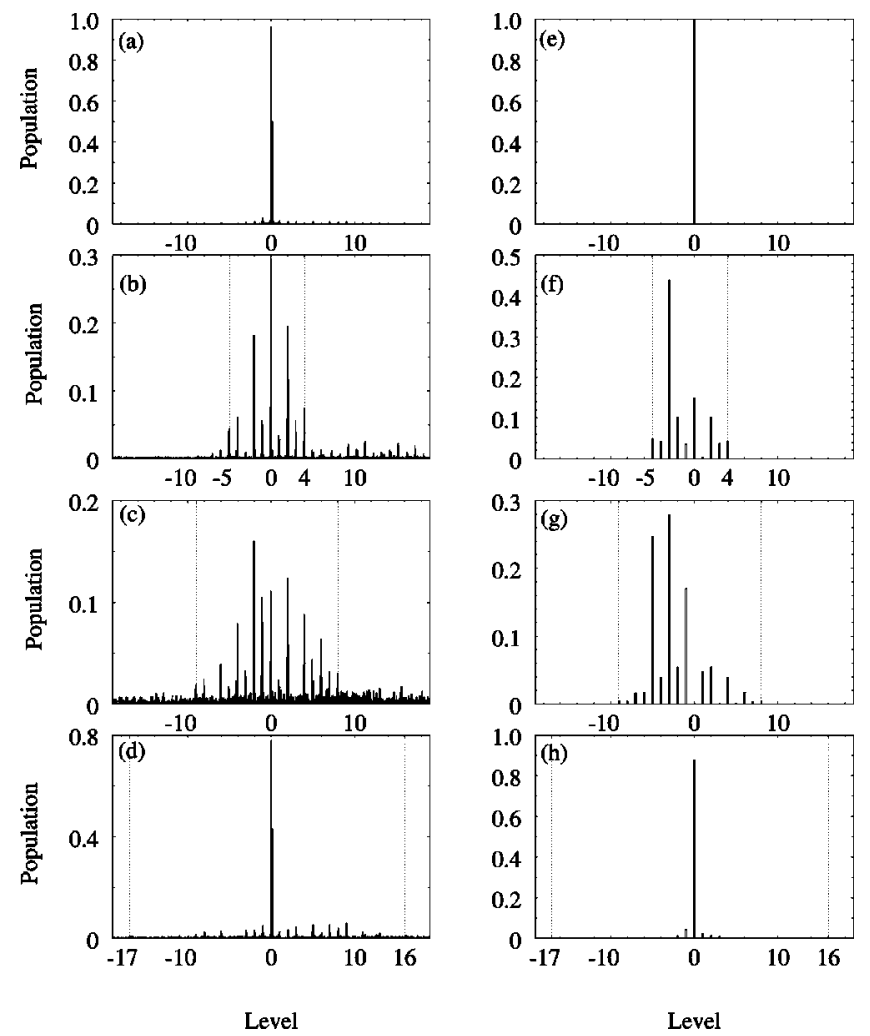

FIG. 7. Experimentally obtained spectral distributions after (a) zero, (b) eight, (c) 16, and (d) 32 columns of crossings, for a semiperiodic grid such that the initial distribution of a single populated level revives after 32 columns of crossings. The transition probability for each double crossing is approximately 0.5 . The corresponding numerical simulations are shown in graphs (e)-(h); they are based on the treatment of the optical Galton board as an array of pointlike beamsplitters in the spectral domain.

optical Galton board, and since residual intracavity birefringence causes an experimental error of about $5 \%$ in measuring $\Delta$, our observation of the recurrences is a rather qualitative one. We performed two types of numerical simulations to support and quantify the experimental observation.

The first type is a simulation based on the naive modeling of each crossing as a pointlike beamsplitter in the frequency domain, as already introduced in Sec. IV. The result of this simulation is displayed in Figs. 7(e)-7(h) and confirms the presence of the recurrences. The second type of simulations is based on numerical integration of Eqs. (10) and (11), and the results shown in Fig. 8 again confirm the recurrences. As mentioned in Sec. IV, no detailed agreement between the experimental and numerical data can be expected since the simulations are based on simplified models of the experiment, and the fluctuations between neighboring level populations depend critically on the exact model. However, interestingly the collective intereference effect, i.e., the recurrences, turns out to be robust (see below) what justifies the presentation of the numerical results.

Since in the experiment the initial distribution revives after 32 columns (Fig. 7) it is expected that further revivals will occur after $64,96, \ldots$ columns. Because the cavity lifetime sets an upper limit to the number of avoided crossings 

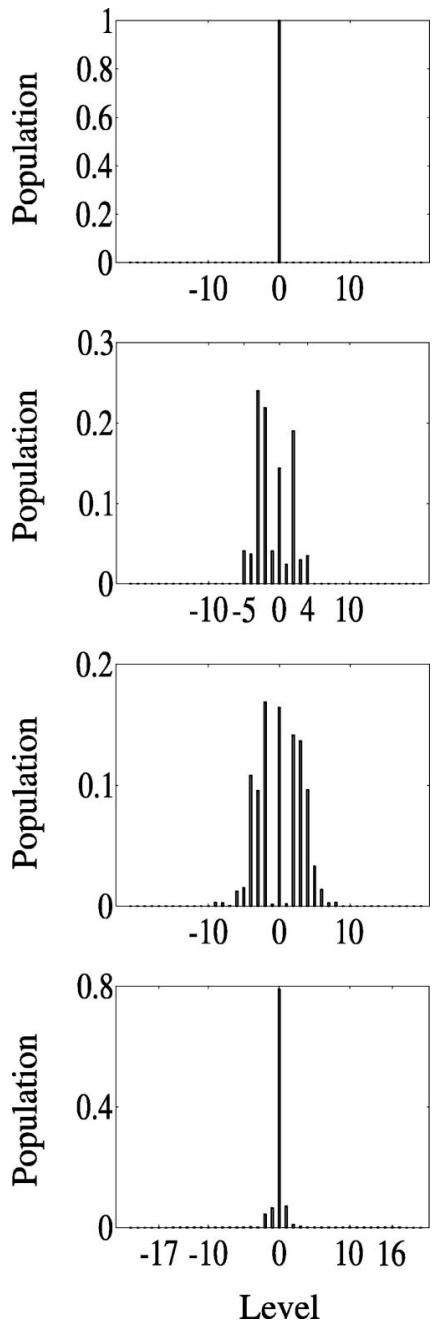

FIG. 8. Simulation of the recurrence phenomenon based on numerical integration of Eqs. (10) and (11).

that can be passed during a single run, these higher-order revivals are out of experimental reach; they were, however, observed in our numerical simulations.

We explored in our numerical simulations the robustness of the recurrences as function of the grid parameters $\alpha$ and $\Delta$. By changing $\Delta$, which effectively changes the transition probability at the crossings in the Galton board while $\Psi$ $=\tau \omega_{\mathrm{FSR}}$ remains constant, we observed that the position of the recurrences remain unchanged up to a transition probability around $0.7-0.8$. Above this transition amplitude the dynamics is so diabatic that most of the population ends up in the outermost levels without ever returning to the initial level population. By changing $\alpha$, which effectively changes both the transition probabilities and the value of $\Psi(\tau$ is proportional to $\alpha$ ), we deduced the following empirical rule: when

$$
\Psi=2 \pi \frac{q}{p},
$$

with $p$ and $q$ integers and relatively prime, recurrences occur after passing $2 p$ columns in the grid of crossings. An explanation for this result will be given in Sec. VI.

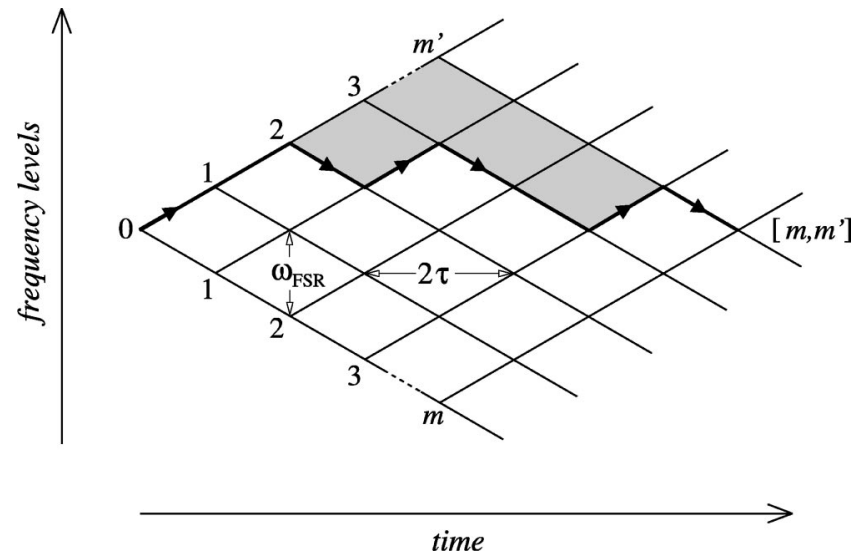

FIG. 9. The grid of crossings resulting from the overlapping of two manifolds of diabatic levels; the horizontal axis represents time. The shaded area corresponds to the dynamical phase associated with the path, marked by arrows, connecting $[0,0]$ to $\left[m, m^{\prime}\right]$.

\section{ANALYTICAL EXPLANATION}

In a recent paper by Harmin $^{1}$ an analytical expression is given for the propagation of a wave through a grid of Landau-Zener crossings [7]. From this paper we extracted the arguments needed to explain the observed recurrences and present them here in a form adapted to our specific optical system. The level structure of the optical system consists of two manifolds of crossing diabatic levels, given by

$$
\begin{gathered}
E_{m}(t)=\frac{\alpha}{T} t-m \omega_{\mathrm{FSR}}, \quad m=0,1,2, \ldots, \\
E_{m^{\prime}}(t)=-\frac{\alpha}{T} t+m^{\prime} \omega_{\mathrm{FSR}}, \quad m^{\prime}=0,1,2, \ldots .
\end{gathered}
$$

We draw a schematic view of the optical Galton board in Fig. 9 in order to clarify the notation, according to which any Landau-Zener crossing is labeled by a pair of coordinates $\left[m, m^{\prime}\right]$. We assume that the dynamics of mode amplitudes consists of a series of isolated pairwise interactions, taking place at each avoided crossing, followed by free evolution during which they acquire only a dynamical phase factor. An avoided crossing can be represented as a $2 \times 2$ unitary matrix,

$$
\left(\begin{array}{cc}
-d & a \\
a & d
\end{array}\right)
$$

with $a$ and $d$ related, respectively, to the adiabatic $A$ and diabatic $D$ transition probabilities

$$
\begin{gathered}
D \equiv d^{2}=\exp \left(-2 \pi \frac{\Delta^{2}}{\alpha T}\right), \\
A \equiv a^{2}=1-D .
\end{gathered}
$$

\footnotetext{
${ }^{1}$ Our experiments were performed before the publication of Ref. [7]
} 
The parameters $\Delta$ and $\alpha$ have been defined in Eq. (8). The phase shift accumulated along an arbitrary path in the array of levels, connecting the initial state $[0,0]$ with the point $\left[m, m^{\prime}\right]$, is calculated with respect to the reference path $[0,0] \rightarrow\left[0, m^{\prime}\right] \rightarrow\left[m, m^{\prime}\right]$,

$$
\Phi_{0}=\int_{0}^{m^{\prime} \tau} d t^{\prime} E_{0}\left(t^{\prime}\right)+\int_{m^{\prime} \tau}^{\left(m+m^{\prime}\right) \tau} d t^{\prime} E_{m^{\prime}}\left(t^{\prime}\right),
$$

where $\tau$ is the time interval between two adjacent crossings. According to this definition the dynamical phase of every path is simply the area between that specific path and the reference one: this amounts to an integer number of cells in the array of avoided crossings with elementary area $\Psi$ $=\tau \omega_{\mathrm{FSR}}$. Note that this quantity $\Psi$ is the same phase as defined in Eq. (12)

One may distinguish between the probability to reach a given point $\left[\mathrm{m}, \mathrm{m}^{\prime}\right]$ from below (along an up going level) or above (following a down going level); to this end we define $A_{m m^{\prime}}^{(\uparrow)}$, and $A_{m m^{\prime}}^{(\downarrow)}$ which represent, respectively, the probability amplitude to arrive at $\left[m, m^{\prime}\right]$ from $\left[m, m^{\prime}-1\right]$ and from $\left[m-1, m^{\prime}\right]$. The evolution from an avoided crossing to the next one can then be summarized by the recursion relations

$$
\begin{gathered}
A_{m m^{\prime}}^{(\uparrow)}=\left[-d A_{m m^{\prime}-1}^{(\uparrow)}+a A_{m m^{\prime}-1}^{(\downarrow)}\right] e^{i m \Psi}, \\
A_{m m^{\prime}}^{(\downarrow)}=a A_{m-1 m^{\prime}}^{(\uparrow)}+d A_{m-1 m^{\prime}}^{(\downarrow)} .
\end{gathered}
$$

The extra exponential factor in Eq. (20) with respect to Eq. (21) takes into account the increased phase difference of the path leading upwards compared to the down going one. These equations, together with the initial condition $A_{00}^{(\uparrow)}=1$, contain the essential information to solve the problem.

Making use of generating functions and an expansion in powers of $d$, as shown in the Appendix, one obtains in the near adiabatic regime $\left(d^{2} \ll 1\right)$ the formal solution

$$
\begin{aligned}
A_{m m^{\prime}}^{(\uparrow)}(\Psi)= & \frac{\Phi_{1}}{2 \pi} \int_{0}^{2 \pi} d x^{\prime} \\
& \times \exp \left\{i\left[2 d \frac{\sin \left(m^{\prime} \Psi / 2\right)}{\sin (\Psi / 2)} \sin x^{\prime}+\left(m-m^{\prime}\right) x^{\prime}\right]\right\},
\end{aligned}
$$

where $\Phi_{1}$ is an overall phase factor. A similar expression holds for $A_{m m^{\prime}}^{(\downarrow)}(\Psi)$. Equation (22) is the integral representation of a Bessel function of integer order $\nu=m-m^{\prime}$ :

$$
\left|A_{m m^{\prime}}^{(\uparrow)}\right|=\left|J_{\nu}\left(2 d \frac{\sin \left(m^{\prime} \Psi / 2\right)}{\sin (\Psi / 2)}\right)\right| .
$$

From the property of Bessel functions

$$
J_{\nu}(-x)=(-1)^{\nu} J_{\nu}(x),
$$

one can predict the periodicity in the probability distribution. The population in $\left[m+\Delta m, m^{\prime}+\Delta m^{\prime}\right]$ is equal to the one in $\left[m, m^{\prime}\right]$, when

$$
\Delta m^{\prime} \Psi=0 \bmod 2 \pi
$$

for example, if $\Psi=2 \pi q / p$, with $p$ and $q$ integers and relatively prime, the recurrences take place after $\Delta m^{\prime}=p$ periods of crossings. Since the order $\nu$ of the Bessel function must be kept constant, the increments in $m$ and $m^{\prime}$ are equal, and the recurrences involve points on the grid which are parallel to the time axis (see Fig. 9). This is in agreement with the experimental and numerical results; the initial population distribution $\left|A_{00}^{(\uparrow)}\right|$ reappears in $\left|A_{p p}^{(\uparrow)}\right|$ after passing $2 p$ columns of crossings (effectively $p$ periods of crossings).

\section{CONCLUDING DISCUSSION}

We have performed optical and numerical experiments in which we studied a wave-mechanical analog of classical diffusion on a Galton board. Our specific system consists of a grid of Landau-Zener crossings produced inside an optical resonator and leads to spectral diffusion of light inside the resonator. Although the system is completely within the domain of classical optics, the observed wave-mechanical dynamics can be described by a Schrödinger-like equantion [see Eqs. (4)-(6)], and could as well be observed in quantum systems with similar (energy-) level structures. The optical system allowed for a study of coherent dynamics of light for a time span as long as $100 \mu \mathrm{sec}$.

The main result is the observation of recurrences of the initial spectral distribution for special values of $\Psi$. Here $\Psi$ $=\tau \omega_{\mathrm{FSR}}$, with $\tau$ the time between two Landau-Zener crossings and $\omega_{\mathrm{FSR}}$ equal to $2 \pi$ times the free spectral range. As the initial distribution we populated a single level. However, since the system is linear it is expected that any arbitrary initial distribution will show recurrences. The requirement for the recurrences to occur after passing $p$ periods in the grid of crossings is (i) that $\Psi=2 \pi q / p$, with $p$ and $q$ integers and relatively prime; and (ii) that the transition probabilities at the crossings are less than about 0.8 . Since any value of $\Psi$ is arbitrarily close to a fraction of two integers that are relatively prime, there will always be such recurrences, provided that the transition amplitude is smaller than about 0.8 . Hence an important conclusion is that the diffusion on a wavemechanical Galton board is in general strongly suppressed compared to classical diffusion.

Our experimental results are consistent with the numerical simulations and with the analytical results based on the theory by Harmin [7]. Note that the theory presented above was performed under the adiabatic assumption, that is, the transition probabilities are assumed to be much smaller than 1. However, from our experimental and numerical result we can conclude that the recurrences persist for transition probabilities up to 0.8 . Qualitatively it is obvious that in the diabatic regime (transition probabilities close to one) the recurrences will have to disappear, since only the outermost levels will have significant populations. It is, however, far from obvious at what value of the transition amplitude the recurrences should vanish. Analytical calculations in the diabatic limit can be found in Ref. [7], and they show that diffusion in the diabatic regime has the special form of narrow peaks in the distribution which move away from the position of the 
initial peak in the distribution function. Further experimental effort is needed to observe this dynamics. The transition from diffusion in the adiabatic regime to diffusion in the diabatic regime is still an unexplored area although it has been suggested that the transition is similar to a phase transition in a two-dimensional Ising model [8].

\section{ACKNOWLEDGMENTS}

The authors thank G. Nienhuis, S. van Enk, P. Törmä, and D. A. Harmin for stimulating discussions on this subject. This work was part of the research program of the Foundation for Fundamental Research on Matter (FOM), and was made possible by the financial support from the Netherlands Organization for Scientific Research (NWO). We also acknowledge support from the TMR Contract Nos. ERB4061PL95-1021 and ERBFMRXCT96-0087.

\section{APPENDIX: GENERATING FUNCTIONS}

By introducing the generating functions

$$
\begin{aligned}
& F_{m^{\prime}}(x)=\sum_{m=0}^{\infty} A_{m m^{\prime}}^{(\uparrow)} e^{i m x}, \\
& G_{m^{\prime}}(x)=\sum_{m=0}^{\infty} A_{m m^{\prime}}^{(\downarrow)} e^{i m x},
\end{aligned}
$$

the system of coupled equations (20) and (21) turns into

$$
\begin{gathered}
F_{m^{\prime}}(x)=-d F_{m^{\prime}-1}(x+\Psi)+a G_{m^{\prime}-1}(x+\Psi), \\
G_{m^{\prime}}(x)=e^{i x}\left[a F_{m^{\prime}}(x)+d G_{m^{\prime}}(x)\right],
\end{gathered}
$$

which can be solved to give

$$
\begin{gathered}
F_{m^{\prime}}(x)=\prod_{k=1}^{m^{\prime}} \frac{e^{i(x+k \Psi)}-d}{1-d e^{i(x+k \Psi)}} \\
G_{m^{\prime}}(x)=\frac{a}{e^{-i x}-d} F_{m^{\prime}}(x) .
\end{gathered}
$$

A Fourier transform leads to the formal solution for the probability amplitude at each site of the grid,

$$
A_{m m^{\prime}}^{(\uparrow)}(\Psi)=\frac{1}{2 \pi} \int_{0}^{2 \pi} d x e^{-i m x} \prod_{k=1}^{m^{\prime}} \frac{e^{i(x+k \Psi)}-d}{1-d e^{i(x+k \Psi)}},
$$

and to a similar solution for $A_{m m^{\prime}}^{(\downarrow)}(\Psi)$.

Every term of the product in Eq. (A7) is a complex number with unit modulus

$$
\begin{gathered}
\frac{e^{i(x+k \Psi)}-d}{1-d e^{i(x+k \Psi)}} \equiv \exp [i(2 \Theta+x+k \Psi)], \\
\Theta=\arctan \left[\frac{\sin (x+k \Psi)}{\cos (x+k \Psi)-d}\right]-(x+k \Psi) .
\end{gathered}
$$

This form is more convenient because it lends itself to an expansion in powers of $d$; in the nearly adiabatic regime $\left(d^{2} \ll 1\right)$ one can retain just the first term

$$
\Theta \simeq d \sin (x+k \Psi) .
$$

Inserting Eqs. (A8) and (A10) into Eq. (A7) leads to the final result given in Eq. (22) (for details, see Ref. [7]).
[1] D. Bouwmeester, N. H. Dekker, F. E. v. Dorsselaer, C. A. Schrama, P. M. Visser, and J. P. Woerdman, Phys. Rev. A 51, 646 (1995).

[2] Quantum Chaos; Between Order and Disorder, edited by G. Casati and B. Chirikov (Cambridge University Press, Cambridge, 1995), pp. 3-55.

[3] F. Haake, Quantum Signatures of Chaos (Springer, Berlin, 1990).

[4] L. E. Reichl, The Transition to Chaos in Conservative Classical Systems: Quantum Manifestations (Springer-Verlag, Berlin, 1992), and references therein.

[5] F. L. Moore, J. C. Robinson, C. F. Bharucha, Bala Sundaram, and M. G. Raizen, Phys. Rev. Lett. 75, 4598 (1995).

[6] U. Stoffregen, J. Stein, H.-J. Stöckmann, M. Kuś, and F. Haake, Phys. Rev. Lett. 74, 2666 (1995), and references therein.

[7] D. A. Harmin, Phys. Rev. A 56, 232 (1997).

[8] P. Törmä, Phys. Rev. Lett. 81, 2185 (1998).

[9] Rydberg States of Atoms and Molecules, edited by R. F. Stebbings and F. B. Dunning (Cambridge University Press, New
York, 1983), Chaps. 3 and 9.

[10] T. F. Gallagher, Rydberg Atoms (Cambridge University Press, New York, 1994).

[11] R. C. Jones, J. Opt. Soc. Am. 31, 488 (1941).

[12] R. J. C. Spreeuw and J. P. Woerdman, in Progress in Optics, edited by E. Wolf (North-Holland, Amsterdam, 1993), Vol. 31, pp. 263-319.

[13] C. A. Schrama, D. Bouwmeester, G. Nienhuis, and J. P. Woerdman, Phys. Rev. A 51, 641 (1995).

[14] L.D. Landau, Phys. Z. Sowjetunion 2, 46 (1932).

[15] C. Zener, Proc. R. Soc. London, Ser. A 137, 696 (1932).

[16] J. E. Bayfield and P. M. Koch, Phys. Rev. Lett. 33, 258 (1974); P. M. Koch, in Chaos and Quantum Chaos, Proceedings of the Eighth South African School in Physics, edited by Heiss (Springer-Verlag, Berlin, 1993).

[17] M. C. Baruch and T. F. Gallagher, Phys. Rev. Lett. 68, 3515 (1992).

[18] S. Yoakum, L. Sirko, and P. M. Koch, Phys. Rev. Lett. 69, 1919 (1992). 